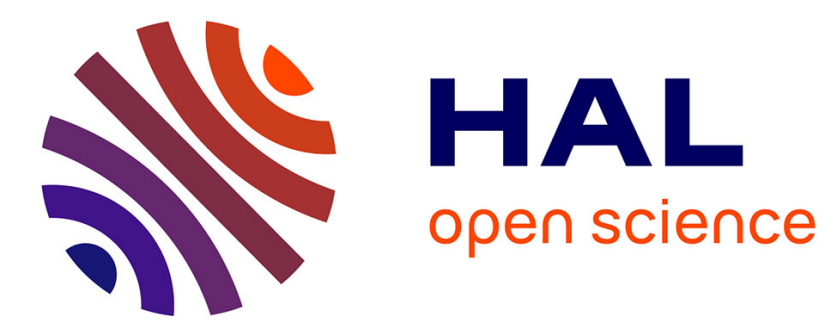

\title{
Les enseignants de droit face à la norme linguistique à l'universite
}

\author{
Marc Debono
}

\section{To cite this version:}

Marc Debono. Les enseignants de droit face à la norme linguistique à l'universite. Le Français Aujourd'hui, 2011, 173, pp.89-101. hal-01376799

\section{HAL Id: hal-01376799 \\ https://hal.science/hal-01376799}

Submitted on 5 Oct 2016

HAL is a multi-disciplinary open access archive for the deposit and dissemination of scientific research documents, whether they are published or not. The documents may come from teaching and research institutions in France or abroad, or from public or private research centers.
L'archive ouverte pluridisciplinaire HAL, est destinée au dépôt et à la diffusion de documents scientifiques de niveau recherche, publiés ou non, émanant des établissements d'enseignement et de recherche français ou étrangers, des laboratoires publics ou privés. 
DEBONO, M. (2011), "Les enseignants de droit face à la norme linguistique à l'université », In : CADET, L. et BISHOP, M.-F. (dirs.), "Ruptures et continuités dans l'acquisition de la maîtrise de la langue à l'école», Le français aujourd'hui, n¹73, pp. 89-101. Disponible sur Cairn.info: http://www.cairn.info/revue-le-francais-aujourd-hui-2011-2-page-89.htm

\section{Les enseignants de droit face à la norme linguistique}

FA n'173 - Ruptures et continuités dans l'acquisition de la maîtrise de la langue à l'école

Marc Debono

Résumé : L'enseignement supérieur du droit, intrinsèquement traversé par les questions de norme, constitue un contexte didactique intéressant pour étudier les fonctionnements normatifs en matière linguistique. L'analyse d'un corpus d'entretiens réalisés auprès d'enseignants de droit à l'Université fait émerger des représentations linguistiques très conservatrices, qui peuvent en partie s'expliquer par l'histoire personnelle des témoins, mais aussi par l'histoire intriquée de la construction des normes juridique et linguistique en France, intrication qui aboutit à considérer leur fonctionnement comme comparable. Cette idée, encore prégnante aujourd'hui, est nettement perceptible chez les deux grands « grammatiseurs » que sont Meigret et Vaugelas. D'un point de vue didactique, elle conduit au normativisme linguistique des formations juridiques, ainsi qu'à la focalisation des enseignements de français juridique (français langue étrangère) sur des dimensions strictement linguistiques.

Mots clefs : norme, enseignement supérieur, représentations linguistiques, droit, formation.

\section{1. « Nul n'est censé ignorer la loi linguistique »}

Depuis 2006, notre système scolaire est fondé sur le principe du " socle commun », consistant à définir «ce que nul n'est censé ignorer en fin de scolarité obligatoire sous peine de se trouver marginalisé » (BOEN n²9 du 20 juillet 2006). Le rappel de ce principe n’a ici d'autre fin que d'attirer l'attention sur la référence au célèbre adage juridique «nul n'est censé ignorer la loi », traduction de l'ancienne formule latine «nemo censetur legem ignorare ». Si le socle commun concerne un ensemble très diversifié de savoirs et de compétences, les dimensions linguistiques y occupent une place importante. Il est dès lors tentant de faire le rapprochement avec ce qu'écrit Bourdieu à propos de l'acquisition de la " langue légitime » : «Nul n'est censé ignorer la loi linguistique qui a son corps de juristes, les grammairiens [...] » (P. Bourdieu, 2001 : 71). Plus généralement, dans son célèbre texte sur « La production et la reproduction de la langue légitime », Bourdieu emploie nombre de termes, d'expressions et de métaphores juridiques : " la sanction juridique du titre scolaire » (idem : 71), " code », « efficacité juridique », « arrêtés » (idem : 76), « fictio juris » (idem : 68), etc.

On peut poser que le juridisme dans la conception de la norme linguistique se manifeste à tous les stades du système d'enseignement français, avec des conséquences formatives diverses. Dans une recherche récente (M. Debono, 2010a), nous nous sommes plus particulièrement intéressé au contexte didactique de l'enseignement supérieur, en allant interroger des enseignants de droit sur leurs représentations linguistiques. L'objet de cette contribution est de présenter une analyse de ces entretiens qui laissent transparaître chez les témoins interrogés 
un attachement, parfois très conservateur, à la norme linguistique. Cet attachement sera utilement mis en perspective historique : l'histoire française des unifications linguistique et juridique montre en effet une forte connivence entre juristes et grammairiens, connivence susceptible d'expliquer la rigidité de la conception de la norme linguistique dans les formations juridiques - et d'expliquer par extension le «linguistico-centrisme» des formations destinées à préparer les étudiants allophones à l'intégration des cursus de droit français.

\section{Représentations linguistiques de quelques enseignants de droit à l'Université}

Les entretiens que nous nous proposons d'analyser ici ont été réalisés entre 2007 et 2008 auprès de sept enseignants-chercheurs (Maîtres de Conférences et Professeurs) de la faculté de droit d'une université française ${ }^{1}$.

\subsection{Une première représentation : l'idée d'un rapport d' « intimité » à la langue}

Si l'on se réfère à ce que disent spontanément les enseignants-juristes de leur « rapport à la langue » (question générale, par laquelle les entretiens commençaient dans la plupart des cas), une constante dans leurs réponses est l'idée d'une grande " proximité » à la langue dans leur pratique, proximité qui serait une particularité du travail du juriste. Certains évoquent cette proximité en termes affectifs : selon $\mathrm{T} 7$, « le BON juriste a un certain AMOUR de la langue française », tandis que pour T3, l'étudiant en droit doit apprendre à " jouer avec la langue, à utiliser la langue, à aimer les mots ». Quant à T1, il qualifie d'emblée ce rapport des juristes à la langue d' « intime ». En schématisant, on peut résumer ce que T1 entend par " intimité » en deux propositions symétriques :

- Le droit, c'est de la langue : lors de l'entretien, il définit en effet spontanément le droit comme étant « un langage », ou encore « une grammaire ».

- La langue, c'est du droit : comme l'exprime T1, travailler sur « le mot », c'est aussi faire du droit. Le témoin rapporte une habitude qui me semble très éloquente à cet égard : les juristes ont coutume de dire que Le Petit Robert (et auparavant Le Littré) constitue « le sixième code », à côté des cinq autres codes « historiques » (civil, pénal, procédures civile et pénale, commerce).

Les deux « systèmes » sont donc comparés dans leur fonction de codification prescriptive : la " grammaire » du droit fonctionne comme la grammaire de la langue, et le « code » juridique est assimilé au code linguistique (d'ailleurs, selon Bourdieu, le mot « code » passe du droit à linguistique : P. Bourdieu, 2001 : 76). Il y a donc une sorte d'assimilation des prescriptions juridiques et linguistiques chez ce témoin, qui ressort clairement de la mise en parallèle très frappante des « fautes » contre le droit et contre la langue. En effet, T1 explique ainsi le degré d'exigence orthographique au concours de l'École Nationale de Magistrature : un juge doit être irréprochable dans son orthographe de par sa fonction, le justiciable étant en droit d'attendre d'être jugé par qui ne commet pas de « faute » contre la langue :

\footnotetext{
${ }^{1}$ Les entretiens sont anonymés : le « $\mathrm{T}$ » (pour «témoin ») est suivi d'un chiffre correspondant à l'ordre chronologique des entretiens. Les conventions de transcription correspondent pour l'essentiel aux règles de ponctuation classiques de l'écrit. Nous avons néanmoins utilisé quelques codes, destinés à faciliter la lisibilité de l'échange (notamment : l'usage des majuscules marque l'insistance, l'emphase sur le mot; les gras marquent un mot ou segment souligné par nous).
} 
$\mathrm{T} 1$ : (prenant le rôle de l'accusé s'adressant au magistrat) Corrigez vos fautes, exprimez-vous dans une langue correcte, ensuite vous me donnerez vos raisons.

La représentation d'une isomorphie des deux systèmes (juridique, linguistique) est un facteur explicatif de la proximité, l'« intimité », l' «amour» de la langue exprimés par les témoins. Pour le dire autrement, la représentation d'un rapport étroit du juriste à la langue est liée à la représentation de la langue comme un système normatif, très comparable au système juridique. L'idée d'une identité de substance des systèmes linguistiques et juridiques peut également expliquer le normativisme affirmé de certains témoins en réaction à la baisse ressentie du niveau linguistique des étudiants en droit.

\subsection{Le risque d'anomie : le thème de la baisse du niveau linguistique des étudiants et la « crise » du français}

Certains des enseignants que nous avons interrogés semblent très sensibles au risque d'anomie linguistique, attribuée au très populaire thème de la «crise de la langue $»^{2}$. Par exemple, sans que cela soit particulièrement recherché, l'entretien avec T3 va porter dans sa quasi-totalité sur la question du "niveau» linguistique des étudiants français en droit. Les propos de $\mathrm{T} 3$ sont très sévères sur ce niveau, et plus particulièrement sur celui des « première année » :

T3 : [...] Et en première année, c'est TOUS ! Quel étudiant ne commet pas, n'écrit pas... avec PLEIN de fautes d'orthographe, c'est BOURRÉ de fautes! Parfois, je vous dis, la copie est ILLISIBLE, on n'arrive pas à comprendre ce qu'il veut faire passer comme message, enfin ce qu'il a appris, ce qu'il a retenu du cours, on comprend rien du tout !

Un peu après, répondant à une question sur la place du critère linguistique dans l'évaluation des travaux de ses étudiants :

T3 : [...] à l'écrit, là c'est CRIANT, hein! C'est vraiment TERRIBLE ! Le niveau d'orthographe, le niveau grammatical, de syntaxe... enfin TOUT, TOUT, TOUT est atterrant.

T4 tient sensiblement le même discours :

E : Est-ce que c'est un critère [le critère linguistique] que vous prenez en compte dans votre évaluation de vos étudiants?

T4 : Non. Euh... honnêtement non, parce que ce serait... ce serait (rires) dirimant. Personne n'aurait la moyenne. C'est pas possible. Mais, je leur dis et ils l'acceptent très bien et ils en tiennent compte. Moi c'est ça qui m'intéresse. C'est pas de les sanctionner là-dessus. Si, bien sûr, en première année vous avez des copies illisibles, vous pouvez pas mettre la moyenne à des copies illisibles.

Une des caractéristiques du thème de la crise de la langue est le sentiment d'une évolution, d'une « baisse du niveau $»^{3}$ :

T3 : [...] nous on laisse passer, on est de plus en plus passoire hein ! On laisse passer de plus en plus de fautes d'orthographe.

E: Il y a une évolution de ce point de vue là ? Vous avez l'impression de laisser plus « passer », d'être moins attentive à l'orthographe ?

\footnotetext{
${ }^{2}$ Sur la popularité de ce thème, voir entre autres : J. Chaurand, $1999: 369$.

${ }^{3}$ Sentiment largement irrationnel, comme l'ont montré C. Baudelot et R. Establet dans leur fameux ouvrage Le niveau monte (1989).
} 
T3 : Moi j’y ai toujours été sensible. Mais autrefois... enfin, quand je dis « autrefois », je suis pas si vieille que ça, mais enfin en à peu près quinze-vingt ans d'activité... j'ai l'impression qu'il y a eu un glissement, en tout cas je retrouve beaucoup plus d'étudiants qui écrivent encore avec des fautes d'orthographe au niveau de la quatrième année qu'auparavant. J'ai l'impression. Il y a quand même là, on va prendre sur quinze ans à peu près hein... sur quinze ans, on a toujours eu des étudiants qui faisaient des fautes en première année, en revanche en licence-maîtrise c'était rare, aujourd'hui c'est fréquent.

Le terme de « glissement » revient dans la suite de l'entretien, T3 évoquant « un glissement et un appauvrissement de la langue et du niveau de français de nos étudiants » qui empêche ces derniers de « franchir les étapes » : l'image du « franchissement» revient également souvent dans son discours, la première "étape » à franchir par l'apprenti juriste étant celle de la langue. On trouve la même idée dans le discours de T4, qui affirme même évaluer le taux de franchissement, faire des "statistiques" (avec des critères très souples) sur le niveau de maîtrise linguistique de ses étudiants :

T4 : Alors j'ai demandé à mes chargés de $\mathrm{TD}^{4}$, en première année (je dis bien en première année, en première année on a beaucoup d'étudiants, on en a 700 ou 800, c'est nous dans l'Université $X$ qui en avons le plus en fait), je leur ai demandé de me dire combien d'étudiants, à leurs yeux, avaient une maîtrise correcte (je dis pas exceptionnelle, mais correcte), du français. La réponse, c'est trente pour cent. C'est-à-dire qu'il y en a trente pour cent qui maîtrisent le français comme on doit le maîtriser en ayant le bac, quoi.

$\mathrm{E}:$ Et vous aviez donné des critères?

T4 : Non, non. J'ai pas donné de critères, je leur ai dit « vous me dites, d'après vous »... et je l'ai fait aussi avec les copies que j'avais, et j'ai trouvé la même chose. Et il se trouve, c'est ça qui est étonnant, que ça correspond (grosso modo c'est pas à un pour cent près, on a pas fait des trucs très précis), ça correspond, que ça correspond à ceux qui ont leur première année. Il y a trente pour cent des étudiants qui ont leur première année : ça correspond à ceux qui maîtrisent le français.

C'est une autre caractéristique du discours sur la crise de la langue que de s'appuyer sur un « ressenti », de ne jamais pousser l'interrogation sur les critères utilisés pour évaluer le niveau («J'ai pas donné de critères, je leur ai dit « vous me dites, d'après-vous »...). Si les critères d'évaluation ne sont pas précisés, le témoin expose néanmoins quelques exemples d'erreurs fréquentes chez ses étudiants :

T4 : [...] Vous voyez, par exemple, j'ai fait passer des oraux toute la journée, donc j'ai des étudiants... j'ai au moins... facilement deux étudiants sur dix qui emploient l'expression « au final». Ben « au final», je suis désolé ce n'est pas français. Le français, c'est « finalement», « en définitive », « en fin de compte », c'est comme on veut, mais c'est pas « au final». «Au final» c'est journalistique. Vous voyez/ donc MOI je leur fais la remarque, je leur dis «ça, c'est pas français ».

Dans les marges des copies annotées par T4 auxquelles nous avons eu accès, le commentaire, péjoratif, de " journalistique » revient souvent : l'équivalence posée entre " pas français » et «journalistique » («[...] ce n'est pas français. [...] c'est journalistique »), témoigne d'une stigmatisation relativement fréquente chez les puristes : le style «journalistique » n'est-il pas l'équivalent actuel des usages linguistiques des « mauvais auteurs », contre lesquels Vaugelas mettait déjà en garde ? Dès le début de notre entretien, à propos des pratiques linguistiques des jeunes enseignants de droit cette fois, $\mathrm{T} 4$ commence sa diatribe contre les « journalistes » :

\footnotetext{
${ }^{4}$ Travaux dirigés.
} 
T4 : [...] les juristes... ceux qui par exemple partent à la retraite en ce moment, ou qui sont partis à la retraite dans les vingt dernières années, étaient des gens qui avaient une formation CLASSIQUE très solide et qui donc avaient une langue, un français TRÈS CLASSIQUE. Bon, ça c'est le premier point... et les juristes de ma génération, bon parce que moi je suis parmi les plus jeunes à la fac, ou des générations immédiatement précédant la mienne, ben il parlent un français BANAL, le français... pas forcément celui de la rue, mais pire que ça, peut-être le français des journalistes, souvent.

Le « français des journalistes » est donc en bas de l'échelle, distingué du français « banal», ou même du français « de la rue », qui semblent préférables au témoin (on soulignera le " pire que ça »). Ces «français » sont bien sûr opposés au «français très classique », celui des enseignants plus âgés, ayant reçu « une formation classique très solide »: la " baisse du niveau » touche donc à ses yeux les étudiants, mais aussi les enseignants. Le propos de T4 est donc très normatif: la distinction entre la «langue » et la «non-langue » (« ce n'est pas français » / " c'est journalistique ») et la stigmatisation des variations (avec la hiérarchisation des différents « français ») sont affirmées avec assurance, et ceci en s'appuyant sur l'exemple d'usages linguistiques pourtant très répandus (« au final»). Un autre exemple d'erreur qui l' «agace » est la tendance à personnifier les abstractions. T4 donne cet exemple, tiré d'une copie annotée : "la société contemporaine assiste à la privatisation de la sécurité des biens et des personnes ». Là encore, nous sommes face à un usage, certes contraire à la norme prescriptive, mais largement répandu.

Le conservatisme linguistique de nos témoins se manifeste donc par une nostalgie du « beau langage », du " français très classique », mais aussi par un attachement au latin : « Moi je suis resté bloquée... au latin! (rires) », dit T3. Cet attachement au latin juridique est parfois moins explicite : T5, sans exprimer de positions conservatrices sur le sujet (son discours est plutôt modéré sur ce point), utilise très naturellement beaucoup de latinismes dans son discours : « le droit romain a été perçu comme ratio scripta, quelque chose d'indépassable "; "c'était contra legem, c'était contre le Code civil! »; "Si vous dites que 'fraus omnia corrumpit', et qu'on va pouvoir annuler une action juridique..."; "ce qu'on appelait les "professions libérales » (artes liberales chez les romains) »; etc.

Un certain conservatisme linguistique amène corollairement chez ces enseignants la perception d'une « crise de la langue ", perception qui, il faut tout de même le noter, est loin de leur être propre. T3 s'appuie d'ailleurs elle-même sur l'actualité (toute relative !) de cette " crise ", en faisant référence à un dossier récent du Nouvel Observateur sur l'illettrisme $\left(\mathrm{n}^{\circ} 2235\right.$, septembre 2007), et conclut ainsi notre entretien en faisant référence aux « débats aujourd'hui sur l'école, sur le niveau d'orthographe, de français... ». On peut légitimement penser que les réactions n'auraient pas été d'une nature très différente si l'on avait interrogé des (ou certains ${ }^{5}$ ) universitaires d'autres filières. Néanmoins, chez les enseignants de droit, la présence de la thématique de la baisse du niveau linguistique est un indice supplémentaire de l'importance accordée à la dimension linguistique de l'activité juridique. Pour le dire autrement, il y a un fort sentiment d'un lien entre la « qualité de la langue » et la « qualité du droit »: la détérioration supposée de la maitrise linguistique pourrait aboutir à une détérioration de la qualité du droit, et c'est pour cette raison que les témoins affichent et revendiquent facilement leur normativisme linguistique.

\subsection{Face à la « crise » : revendication d'un conservatisme puriste}

\footnotetext{
${ }^{5}$ Il ne s'agit pas, bien évidemment, d'homogénéiser les universitaires selon leur appartenance disciplinaire. Par exemple, si nos entretiens ont révélé des représentations partagées, il y a également beaucoup de diversité dans les opinions émises par les témoins.
} 
La « réaction » face à la crise de la langue, amène une auto-catégorisation de T3 comme « puriste extrémiste »:

T3 : [...] Alors qu'est-ce qu'on peut faire à notre niveau ? On va pas recommencer le primaire, enfin c'est pas possible ça! Moi je suis beaucoup plus rigoureuse, enfin rigoureuse... extrémiste : c'est-à-dire que les étudiants s'y mettent par eux-mêmes, font l'effort, bouquinent, reprennent quelques règles de grammaire de base en aidant un petit frère ou une petite sœur, pourquoi pas... ou en donnant des cours du soir, ça leur permettra d'apprendre eux aussi le français ! (rires) Mais c'est pas à nous à le faire !

Un peu plus loin, on retrouve cette auto-catégorisation alors que la discussion porte sur les innovations terminologiques et les emprunts aux autres langues :

T3 : [...] moi je vous dis, je suis d'un conservatisme épouvantable... quand je vous dis je suis très classique, en réalité je suis très rigide là-dessus (rires). J'en reste à une terminologie plutôt franco-française, et dans la mesure... pourtant si, si, $\mathrm{j}$ 'intègre aussi maintenant des mots... enfin, je l'utilise pas, mais comme « questionnement» ou autre... enfin, non, moi je suis assez classique, hein, quand même. Ça m’agace. Ça m'agace. Très vite !

Il y a donc chez T3 une récurrence de l'affirmation d'un militantisme «puriste», " conservateur », " extrémiste » en matière de langue. Cette attitude est présentée comme «positive» dans le domaine juridique : pour T3, l'étudiant en droit, futur juriste, se doit d'être « rigoureux » (mot employé et décliné abondamment lors de l'entretien) dans l'emploi de la langue : " purisme », "rigidité » et « conservatisme » sont dès lors présentés comme des qualités. On trouve une auto-catégorisation similaire chez T4, même si elle est plus nuancée :

T4 : [...] vous avez des gens qui sont des militants de la langue française, $\mathrm{j}$ 'en connais, et qui donc vont avoir (et j'avoue que parfois j'en fais partie), vont avoir la volonté de maintenir un français classique, et surtout tout d'abord correct.

Le témoin, qui, nous l'avons vu, commence par exposer une différence générationnelle dans le rapport des juristes à la langue (voir plus haut), finit par s'identifier au groupe "normatif» (avec, tout de même, la petite nuance du «parfois »), même s'il n'appartient pas à la génération d'enseignants censée selon lui employer le «français classique». Cette identification " militante » au groupe d'enseignants normatifs semble également être le cas des chargés de travaux dirigés (TD) de T4. À propos des corrections linguistiques portées sur les copies d'étudiants, il affirme que "plus ils [ses chargés de TD] sont jeunes, plus ils sont violents, durs ». Mais cette violence est finalement jugée justifiée :

$\mathrm{E}$ : Oui, parce que la question de l'expression, de l'orthographe, sont des questions très sensibles qui peuvent produire des réactions... Alors vous me parliez des réactions de vos chargés de TD qui réagissent parfois excessivement...

T4 : Pas excessivement! Moi je dis pas excessivement! Parce que sur le fond, ils ont RAISON. Ils réagissent DUREMENT. Mais ça veut pas dire que c'est excessif.

E : Mais vous expliquiez que vous étiez obligés un peu de... de les calmer/

T4 : Ben sur la forme... sur la forme des fois ${ }^{6}$. Mais calmer... sans non plus exagérer quand même. Sur le fond, je suis d'accord avec eux. Simplement je dis que c'est pas la peine d'en

\footnotetext{
${ }^{6}$ L'expression «au final», prise par ce même témoin comme exemple du style «journalistique » de ses étudiants, est-elle plus problématique que la locution «des fois», donnée comme «populaire»par le dictionnaire Le Robert (1996) ? Preuve que la perception de la conformité de sa propre pratique à la norme linguistique «légitime» peut différer des réalisations effectives, comme Labov a pu le montrer dans ses différentes enquêtes (1976), et comme l'ont mis en évidence en France N. Gueunier, E. Genouvrier et A. Khomsi (1978), comme le montre aussi, dans un contexte universitaire francophone, l'étude de G. Ledegen sur les
} 
rajouter. Vous voyez, c'est pas la peine... parce qu'il peut y avoir une frontière... assez étroite entre la remarque et l'humiliation. L'humiliation ça sert à rien, c'est pas pédagogique, bon le type il est nul, il est nul, ça sert à rien de lui mettre encore... enfin, ça sert à rien. Par contre quelqu'un qu'on suit et qui fait toujours les mêmes erreurs, au bout d'un moment, il faut taper un peu plus fort. Mais en première année non, ça sert à rien. Moi je dis, c'est sur la formulation : ça sert à rien de se moquer d'eux. Bon, quand je mets journalistique, c'est une forme de moquerie, mais bon, c'est modéré. Des fois ils se défoulent un peu (rires). Non mais c'est vrai!

Même si le témoin dit limiter la "violence » en modérant le ton des remarques de ses chargés de TD, il considère que c'est en définitive un "service rendu », en particulier pour les concours (on retrouve la même idée chez T1).

\subsection{Purisme, conservatisme et histoire personnelle}

Si une certaine «rigueur» quant à l'utilisation de la langue est jugée indispensable par l'ensemble des témoins, il n'en va pas de même pour l'affirmation conservatrice et puriste. Il est intéressant de constater que les prises de positions les plus fortes sur la « qualité » de la langue, sur la baisse du niveau émanent de deux témoins (T3 et T4) dont l'histoire personnelle révèle que la norme centrale ne leur a pas été «donnée », mais qu'ils l'ont acquise au prix d'efforts conséquents.

T3 fait en effet une allusion intéressante à son parcours personnel, qui éclaire sa posture " puriste extrémiste » affirmée avec insistance, et montre un rapport complexe à la langue. On peut retracer, à travers ses propos, un cheminement, relativement courant (Labov, 1976), d'une difficulté initiale laborieusement vaincue, à ce normativisme intransigeant.

T3 : Oui. Bon alors, là aussi, malheureusement, il évolue... mais bien sûr, bien sûr ! Et moi je me souviens être arrivée... j'avais passé mon bac dans le Midi, je suis arrivée à Paris II, j'étais SUBJUGUÉE par la MAITRISE du langage, de l'ÉLOCUTION, c'était FORMIDABLE. Formidable. J'ai eu une FASCINATION vraiment. Je parlais très très mal, et j'ai eu un goût, un appétit, si vous voulez, d'essayer de bien parler. J'écrivais pas trop mal mais je parlais très très mal, j'avais un très mauvais oral. Et... (rires) pour devenir enseignante, il fallait que je passe le cap, sinon c'était difficile! Et eux, m'en ont donné le goût, l'envie. Et m'ont montré toute l'importance et toute la séduction qu'il peut y avoir aussi dans l'utilisation du vocabulaire, des concepts, la maîtrise d'une matière... Après, ça peut devenir une séduction, donc c'est pour ça que le côté esthétique est très important. Et il y a des mots qui sont moches : «DÉSENGORGER Paris », c'est très laid (rires). "Rééquilibrer les pouvoirs au profit de la province », c'est tout de suite beaucoup plus beau. Alors évidemment, c'est toute une phrase... mais il y a des mots qui sont laids !

L'insécurité linguistique passée est ici mise en récit pas le témoin : loin de produire de l'indulgence, de la souplesse face à la variation linguistique, cette histoire personnelle en forme de parcours initiatique (« il fallait que je passe le cap »), conduit le témoin à juger très catégoriquement de la "beauté » ou de la «laideur» de certains mots et expressions. On retrouve une trace de cette insécurité originelle quand T3 parle de sa propre orthographe qui serait « menacée » par les « inepties » des étudiants :

T3 : [...] Enfin, moi je travaille beaucoup avec le dictionnaire, hein ! À force de lire des inepties, je finis par douter de mon orthographe... de plus en plus d'ailleurs, j'ouvre le dictionnaire, je suis de moins en moins sûre de moi (rires). On PERD son orthographe quand on lit des fautes tout le temps!

pratiques et représentations linguistiques des étudiants de filières littéraires : Le bon français. Les étudiants et la norme linguistique (2000). 
Quant à T4, d'origine italienne, il dit être locuteur de «dialectes » italiens ainsi que du provençal. Mais ce multilinguisme est loin d'être synonyme de tolérance vis-à-vis des différents usages du français: nous avons vu qu'il n'accepte que très peu la variation « interne » (le « français journalistique » constituant pour lui un puissant repoussoir), et que ses propos sont souvent hypernormatifs. Pourtant, s'il se catégorise comme militant du «français correct», il ne se considère pas du tout comme un "défenseur » de la langue française sur le plan international ${ }^{7}$. Il critique par ailleurs la politique linguistique de « l'État jacobin » (selon lui, l'éradication passée des parlers locaux et la " protection » actuelle du français relèveraient d'une même logique), tout en restant attaché à la norme centrale, au français standard.

On notera par ailleurs que cet hypernormatif, affirmant son attachement militant à la « correction » linguistique, n'hésite pourtant pas à utiliser un français parfois assez éloigné du standard : les expressions « ferme ta gueule !» (attribuée à un énonciateur autre que lui) et «on s'en fout», ou le mot «conneries», employé à l'endroit de deux étudiants qu'il interpelle sur leurs erreurs orthographiques récurrentes :

T4 : [...] Et avec ces deux là, le fait que je dise une fois, deux fois, trois fois devant tout le monde : «oh ! c'est quoi ces conneries ? », sur l'orthographe, au bout d'un moment, ils sont venus me voir.

L'analyse discursive fait donc apparaître, chez T4, des représentations et pratiques linguistiques dénotant un rapport complexe à la norme du français standard, que son histoire personnelle contribue en partie à éclairer. Et il est tout à fait remarquable de constater que l'expression de positions radicales sur la nécessaire correction linguistique des juristes émane des témoins qui nous ont fait part de leur propre « histoire linguistique », histoire marquée dans les deux cas par un éloignement originel de la norme du français standard, norme par la suite survalorisée.

\section{Essai de synthèse : représentations, histoire et conséquences didactiques}

Les enseignants de droit interrogés accordent donc une importance particulière à une langue normée, stabilisée, garantie de sécurité et de rigueur, et ont comme souci formatif de lutter contre son affaiblissement ${ }^{8}$. Ce normativisme n'est peut-être pas spécifique aux juristes, et on peut penser qu'il est partagé par nombre d'universitaires dans d'autres secteurs des sciences humaines et sociales. Néanmoins, la fréquence de sa réapparition dans les entretiens menés nous semble significative ; et plusieurs théoriciens du droit confirment l'influence particulière d'une conception normative de la langue sur l'épistémologie de leur discipline. C'est par exemple le cas de R. Dworkin, pour qui la connaissance juridique est marquée par ce qu'il nomme la « piqûre du dard sémantique » :

\footnotetext{
${ }^{7} \mathrm{Ni}$ des langues régionales d'ailleurs : le témoin, locuteur d'une langue régionale, prend ses distances vis-à-vis du militantisme régionaliste en matière linguistique :

$\mathrm{E}:$ Et donc la protection des langues régionales...

T4 : (soupir) On s'en fout. Quand je dis on s'en fout, c'est pas... je pratique moi-même une langue régionale, donc c'est pas... c'est pas par mépris, c'est que ça n'a pas besoin d'être institutionnalisé, je dirais même au contraire.

${ }^{8}$ Souci qu'ils attribuent d'ailleurs volontiers au chercheur-linguiste les interrogeant : l'enthousiasme (que nous avons pu constater) de ces enseignants à parler des questions de langue peut en effet être lu comme l'expression d'une empathie transdisciplinaire du juriste pour le linguiste catégorisé comme "grammairien normatif», « juriste de la langue » pour reprendre l'analogie bourdieusienne (P. Bourdieu, 2001 : 71).
} 
«J'appellerai cet argument qui a fait un tel ravage dans la philosophie du droit la piqûre du dard sémantique. Les gens en sont victimes quand ils ont une certaine représentation $d u$ fonctionnement $d u$ langage. Ils pensent que nous pouvons parler intelligemment entre nous si - et seulement si - nous acceptons tous de suivre les mêmes règles quand nous décidons de l'usage de chaque mot [...]» (R. Dworkin, 1985 : 106 ; nous soulignons).

L'image de la « piqûre » est bien sûr très ironique chez R. Dworkin, qui considère comme une contamination pernicieuse cette représentation du fonctionnement du langage : considérer que ce n'est qu'en établissant une norme linguistique susceptible de fixer une fois pour toutes le sens des signes linguistiques que l'on peut garantir la sécurité du droit est, pour R. Dworkin, une illusion. Illusion bien sûr, mais la tentation, qualifiée par A. Supiot (tout aussi critique que R. Dworkin), de "prêt-à-penser» fondamentalement erroné, reste forte dans un domaine préoccupé par la sécurité juridique. A. Supiot met en parallèle les représentations du droit et de la langue : le droit est considéré par la doxa comme « une pure technique, une pure affaire de convention(s) », et de la même manière "[1]a langue ne serait qu'affaire de signaux échangés entre des émetteurs et des récepteurs» (A. Supiot, 2003 : n.p.). Peut-on considérer que la langue fonctionne ainsi ? Les témoins interrogés lors de nos entretiens semblent répondre à l'unisson par l'affirmative : s'entendre sur le signal, c'est sécuriser le sens. Mais on aurait tort de voir, dans la recherche d'une sécurisation formelle du sens, l'unique cause d'une recherche de clarté, cohérence, transparence, complétude du langage (juridique). Ces exigences ne sont "pas seulement affaire de logique formelle », elles "dérivent d'une fascination de l'unité » chez les juristes : unité du code, unité de la volonté de l'auteurlégislateur, etc. (J. Lenoble et F. Ost, 1980 : 114).

Il est intéressant de noter que ces représentations linguistiques prennent racine dans une histoire linguistique où les questions de langue ne sont jamais très éloignées des questions juridiques. Nous ne pouvons ici évoquer que de manière allusive la cohérence historique des codifications juridique et linguistique ${ }^{9}$, motivées par le projet d'édification de l'État-nation, et qui interviennent sensiblement au même moment en France : la «fièvre codificatrice » en droit coïncide en effet peu ou prou avec la période d' " outillage » et de codification de la langue française. Le XVI ${ }^{\mathrm{e} m e}$ siècle constitue une période charnière de ce double processus : les grandes opérations du processus de grammatisation (S. Auroux, 1992 et 1994) de la langue française commencent en effet à cette époque ${ }^{10}$, et c'est également lors de ce siècle que naissent nombre de projets de codification du droit, lesquels ponctueront ensuite les XVII $^{\text {ème }}$ et XVIII ${ }^{\text {ème }}$ siècles jusqu'à l'achèvement napoléonien ${ }^{11}$. C'est donc bien au XVI ${ }^{\text {ème }}$ siècle que s'organisent conjointement la construction de la langue française et celle du droit français, qui, auparavant, étaient loin d'être des réalités uniformes : droit du pouvoir royal et mosaïque coutumière, langue française/langue de la cour et mosaïque des parlers régionaux. S'il est vrai que le $\mathrm{XVI}^{\text {ème }}$ siècle est un siècle durant lequel la réforme touche de nombreux secteurs de la vie sociale (poids, monnaies, mesures, langue, droit, religion, etc.), montrant une volonté politique d'accroissement du contrôle social, la coïncidence des poussées uniformisatrices juridique et linguistique n'est pourtant pas anodine : le lien entre les deux réformes est étroit. Si les opérations de grammatisation encadrent étroitement l'Ordonnance de Villers-Cotterêts, édictée par François $\mathrm{I}^{\text {er }}$ en 1539 (qui impose la langue française dans la vie juridique), et si les grandes opérations de codification juridique suivent de près la

\footnotetext{
${ }^{9}$ Pour de plus amples développements, voir : M. Debono, 2010a.

${ }^{10}$ Voir la liste des grammaires qui paraissent à cette époque établie par Auroux, 1992, II : 361-367.

11 Avant de trouver une forme d'aboutissement avec le Code civil de 1804, l'unification du droit français a longtemps, et avec une constance remarquable, été « rêvée» par les monarques français (A. Rigaudière, 2004). « Rêve royal » car, comme pour le projet linguistique, il ne se réalisera que tardivement, bien après la fin de l'ancien régime.
} 
codification linguistique, c'est que l'une rendit possible l'autre (la grammatisation de la langue permet l'unification du droit), et inversement (le droit unifié en français rend nécessaire la création d'outils linguistiques fiables). Autrement dit, si la stabilisation de la langue constitue le socle du droit unifié, l'inverse est également vrai : les vastes réformes juridiques initiées au XVI ${ }^{\text {ème }}$ siècle vont servir de cadre au processus de grammatisation.

L'intrication des deux processus d'édification normative est donc complexe. Deux exemples marquants permettent de mieux en prendre conscience : les théories juridiques ont en effet profondément marqué les deux grandes figures du processus de grammatisation en France que sont Louis Meigret et Claude Favre de Vaugelas. Le premier, formé au droit, prend pour modèle de sélection des usages linguistiques le processus de fixation des usages juridiques dans les recueils de coutumes, lequel se fait sensiblement au même moment (D. A. Kibbee, 2002 et 2003) : la grammaire qu'il publie en 1550, imprégnée de terminologie juridique, est formellement et conceptuellement marquée par cette influence. Quant au second, élevé dans une famille d'illustres juristes, il semble transposer, dans le domaine linguistique, le grand débat juridique de l'époque entre la conception coutumière d'un droit naissant de l'usage, et la conception rationaliste et universaliste du droit naturel. Là encore, l'influence se ressent dans l'écriture, les Remarques sur la langue françoise (C. Vaugelas, 1934 [1647]) étant truffées de termes et d'expressions juridiques (H. Weinrich, 1989). Les théorisations linguistiques des deux grands grammatiseurs sont donc inséparables des conceptions juridiques qui avaient cours aux XVI ${ }^{\text {ème }}$ et XVII ${ }^{\text {ème }}$ siècles en Europe. Les liens entre grammairiens et juristes ne sont d'ailleurs pas propres à la situation française, comme le montre une autre figure importante de l'histoire des idées linguistiques : Jacob Grimm, fondateur de la grammaire historique et comparée, fut également un éminent juriste au sein du courant de l'École historique $d u$ droit, et ses conceptions juridiques et linguistiques présentent de notables convergences (cf. A. Dufour, 1974).

L'intérêt des grammairiens/linguistes pour la logique juridique est donc sensible, et l'histoire intriquée des unifications juridique et linguistique conduit à double monisme (le «droit» et la " langue ») de nature à expliquer la prégnance, au sein de la communauté des juristes contemporains, d'une représentation du fonctionnement de la «langue » à l'image du fonctionnement $\mathrm{du}$ 《droit», « objet» dont l'idéale codification (finitude, complétude, fermeture, totalité) est censée réduire le risque d'équivoque en rejetant toute variation.

Les représentations des enseignants interrogés, inscrites dans une longue histoire linguisticojuridique, ne sont pas sans conséquence didactiques : si les enseignants-juristes attachent une grande importance à la formation linguistique des étudiants, celle-ci est conçue de manière très normative, et le principal conseil donné aux étudiants est de prendre un «code » (Le Robert, "sixième code " des juristes selon T1, ou encore : Bled, Bescherelle, etc.). Ceci n'est sans doute pas étranger au fait que les formations en $\mathrm{FOU}^{12}$ juridique sont souvent très linguistico-centrées, et ne laissent que peu de place au développement des compétences interculturelles / de médiation, pourtant essentielles à la bonne intégration socio-universitaire des apprenants étrangers venant suivre un cursus de droit en France (M. Debono, 2010b et 2010c).

Marc Debono

EA 4246 DYNADIV

Université François-Rabelais, Tours

\footnotetext{
${ }^{12}$ Français sur objectifs universitaires, domaine émergeant relevant de la didactique du français langue étrangère.
} 


\section{Bibliographie}

AUROUX, S. (dir.) (1992), Histoire des idées linguistiques, T.I « La naissance des métalangages en Orient et en Occident», et T.II « Le développement de la grammaire occidentale », Liège, Mardaga.

AUROUX, S. (1994), La Révolution technologique de la grammatisation. Introduction à l'histoire des sciences du langage, Liège, Mardaga,.

BAUDELOT, C. et ESTABLET, R. (1989), Le niveau monte, Paris, Seuil.

BOURDIEU, P. (2001), Langage et pouvoir symbolique, Paris, Seuil.

CHAURAND, J. (dir.) (1999), Nouvelle histoire de la langue française, Paris, Seuil.

DEBONO, M. (2010a), Construire une didactique interculturelle du français juridique: approche sociolinguistique, historique et épistémologique, Thèse, Université de Tours.

DEBONO, M. (2010b), «De l'intérêt de l'herméneutique pour repenser l'interculturel en classe de français juridique », dans P. Blanchet et D. Coste (dirs.), Regards critiques sur la notion d' 'interculturalité'. Pour une didactique de la pluralité linguistique et culturelle, Paris, L’Harmattan, coll. « Espaces Discursifs », pp. 149-172.

DEBONO, M. (2010c), «L'apport d'une approche culturelle du français juridique en termes d'intégration des étudiants étrangers », dans L. Cadet, G. Goes et J.-M. Mangiante, Langue et Intégration. Dimensions institutionnelle, socio-professionnelle et universitaire, Pieterlen, Peter Lang, coll. « GRAMM-R ».

DUFOUR, A. (1974), «Droit et langage dans l'École historique du Droit», Archives de Philosophie du Droit, $\mathrm{n}^{\circ}$ 19, pp. 151-180.

DWORKIN, R. (1985), « La théorie du droit comme interprétation », Droit et société, n¹, pp. 99-114.

GUEUNIER, N., GENOUVRIER, E., et KHOMSI, A. (1978), Les Français devant la norme, Paris, Honoré Champion.

KIBBEE, D. A. (2002), «L'autorité de l'État et l'autorité linguistique », Histoire Épistémologie Langage, vol. 24, n², pp. 5-27.

KIBBEE, D. A. (2003), « Louis Meigret, Lyonnais, et les politiques de la langue à la Renaissance ", dans G. Defaux (dir.), Lyon et l'illustration de la langue française à la Renaissance, Lyon, Presses de l’École Normale Supérieure, pp. 63-76.

LABOV, W. (1976), Sociolinguistique, Paris, Éditions de Minuit.

LEDEGEN, G. (2000), Le bon français. Les étudiants et la norme linguistique, Paris, L'Harmattan. 
LENOBLE, J. et OST, F. (1980), Droit, mythe et raison. Essai sur la dérive mytho-logique de la rationalité juridique, Bruxelles, Publication des facultés Universitaires de Saint-Louis.

RIGAUDIÈRE, A. (2004), «Un rêve royal Français : L'unification du droit », Comptes rendus des séances de l'année, Paris: Académie des inscriptions et belleslettres, $n^{\circ} 4$, pp. 1553-1567.

SUPIOT, A. (2003), «Les enjeux linguistiques de la langue juridique », Les Actes de la DESCO, actes du colloque Les contenus culturels dans l'enseignement scolaire des langues vivantes, 4 et 5 décembre 2003, n.p.. URL : http://eduscol.education.fr/cid46257/les-enjeuxlinguistiques-de-la-langue-juridique.html (consulté le 15 février 2010).

VAUGELAS, C. (Favre de) (1934), Remarques sur la langue françoise, fac simile de l'éd. originale de 1647, Paris, Droz.

WEINRICH, H. (1989), «Vaugelas et la théorie du bon usage dans le classicisme français », dans H. Weinrich, Conscience linguistique et lectures littéraires, Paris, Éditions de la Maison des Sciences de l'Homme, pp. 189-218. 